\title{
Health App User Experience Analysis Research: Focusing on the Step Count App
}

\author{
SeungMin Lee \\ Namseoul University, Cheonan, Korea \\ mini0920@nsu.ac.kr
}

\begin{abstract}
Due to the increasing interest of people in health and life sports, health is a major content in various media. This background and development of information technology has led to the release of hundreds of thousands of mobile health applications. Nevertheless, studies evaluating the user experience for mobile app usability and app success are insufficient. Therefore, this study aims to describe the phenomenon and identify problems and improvements by comprehensively exploring the user experience of the step count app of users in their 20s.FGI was performed to grasp the rich empirical data and satisfaction level of users in detail. FGI is a research method conducted by exchanging perceptions and experiences on subjects and cases according to the purpose of the research. As a result of the study, it was found that it is necessary to improve the level of playfulness of the step count app, various additional functions, and reliability.
\end{abstract}

Keywords: Step count app, mHealth, FGI, UX

\section{Research background and purpose}

Health is being treated as a major content in various media due to growing people's interest in health and living sports. In particular, the trend of managing health data using various smart devices is spreading among young people in their 20s and 30s. In addition, smartphones account for the largest portion of the routes they receive health services [1].

Mobile app-based health management can suppress the increase in national health care costs and efficiently promote public health [2]. Therefore, it can be the best strategy that can efficiently promote the health of the people. For this, it is very important to analyze the user experience level and satisfaction level of the app to understand the needs that the user has for the step count app.

In previous studies on health apps, there were studies such as "Analysis of factors affecting acceptance of mobile health apps" and "Relationship between app service quality measurement and continuous use intention". This research is mostly limited to quality of service and use intention. However, research on step count apps, especially user experience for step count apps and user evaluation for app success, is very scarce.

In Therefore, this study aims to improve the user experience by comprehensively exploring the user experience of the step count app of users in their 20s and describing the phenomenon and identifying problems and improvements.

Article history:

Received (February 13, 2020), Review Result (April 2, 2020), Accepted (May 6, 2020) 
The research method consisted of FGI. This study was judged to be appropriate for FGI because it focused on deriving richer strategies by describing and examining the meaning of the user's vivid experiences rather than focusing on interpreting or analyzing objective statistical data.

\section{Research scope and method}

This study conducted an investigation and analysis of users. To propose guidelines for step count apps that are easily accessible to college students among health-related apps.

For analysis evaluation, we selected CashSlides, FreeManboki, and Manbogea apps. As of the end of November 2019, Play Store ranked 1st to 3rd in the number of step count apps usage. The experiment participants were college students in their 20s, who are relatively high in understanding and using the app, and were limited to 4 males and 6 females.

The research method was made up of FGI. The FGI lasted about an hour on December 6, 2019, was recorded with a voice recorder, and the interviews were summarized and described by a research assistant. Five days before FGI was implemented, three step count apps were installed and tried. After that, the experiment participants responded freely to the experiences they felt while using the step count app through FGI, and the interview contents summarized the advantages/disadvantages in terms of content and interface.

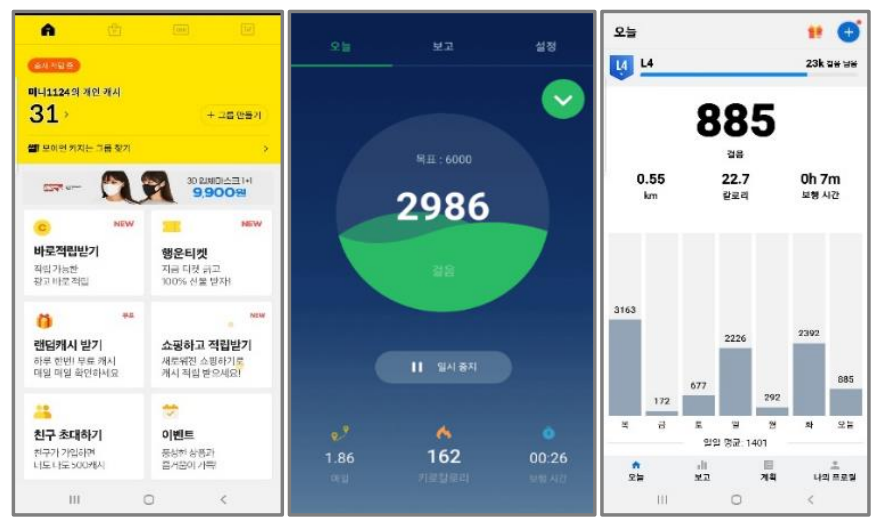

Figure 1. Various step count apps

\section{Results}

\subsection{Result of cash slide users3.1.1 content aspect}

\subsubsection{Content aspect}

\section{It's not just measuring steps, it's motivating by various factors that make it fun.}

The app is connected to stores, so I could buy things with steps that I accumulate, which is great. I think this is great not only to have sense of accomplishment but also to build memory because today's steps can be recorded along with photos. (U2)

By using this app, I could receive points that can be used to buy things, which motivates me. I can earn points by inviting friends, and I can share the points with friends. It is good to compare steps with each other. (U1)

Photos of today's walking record information to keep. The information is recorded in the photos automatically to share on Instagram or gallery, which is very convenient. (U7) 


\section{Excessive advertising makes use uncomfortable.}

It is difficult to distinguish advertisements from others. The app says we can have benefits by earning cash, but I didn't feel like it is benefit because I had to watch too many advertisements. (U3)

Whenever I tried to go back to the pedometer after viewing the benefit or event information, the advertisement appeared and was inconvenient, and it was inconvenient to obtain the desired data. (U9)

There are too many advertisements. We have to use the earned cash at the stores, but the stores are located too far below, which is inconvenient. It is understandable to see advertisements to earn cash, but it is not convenient to see advertisements popped when I start the app.(U7)

\subsubsection{Interface aspect}

\section{Navigation is intuitive and easy to use.}

This is my favorite app among the three. It is easy to watch, and there is no problem in operation or touch. The images of items shown as Gifticon or points that enable to purchase are well organized in high quality. (U1)

It doesn't have separate menu icons, but there are such menus on the main page in the banner form as 'Invite friends', 'Potencube' and 'Immediate saving'. The operation method of the menus is intuitive and easy to see at a glance. The menus are arranged in the order of importance, which makes it easy to use. (U2)

The organization is simple, and its neat design is easy to see, so information search is very simple. Those who use the app for the first time can understand easily. It is processed smoothly without difficulty. In a ward, it is very neat. (U4)

It is divided into a step measurement screen and a shopping screen, so it is difficult and inconvenient to move.

It was inconvenient to find stores and setting because they are located at the bottom. I could check my steps and cash at the main page, but I didn't figure out how the steps and cash are calculated. It was difficult to find the setting to confirm my account information. The absence of menus on the main page is also inconvenient. (U2)

It sometimes takes a while to load locked screen. When I entered the app not pressing notification button, I didn't know where to find the number of steps. (U3)

It is unusual to enter the pedometer and shopping menu. I could enter the pedometer by touching the notification alarm on the smart phone, and for shopping, I had to click the app.When going to the shopping from the pedometer, or vice versa, it is difficult to find easily. It is difficult to express clearly due to my smart phone's dull touch, but it was sometimes recognized with touch if the slide is done quickly. (U6)

\subsection{FGI result of free pedometer app user}

\subsubsection{Content aspect}

It is an app that is faithful to the simple and clean basics without unnecessary functions.

The main page shows today's steps and targeted steps, walking distance and time along with calories consumed. The item 'Statistics' shows my steps on a weekly and monthly basis. The item 'Health' calculates the number of glasses of water, change of weight and BMI, so I 
could check my health state. In addition, the walking time for calories consumed could be checked, along with the process of achieving the goal. (U8)

When I touch the menu button, the sharing menu comes up, so I can share my steps with other apps or messages. Since it is very simple and basic pedometer app without no unnecessary functions, I could concentrate on exercise. The operation methods and procedures are very simple and easy. (U2)

What I really like is the establishment of plan that I could see to make health plan. The menu is very suitable for the term 'plan' like losing and maintaining weight. It also has only four necessary plans, so it is not that complicated. However, those who want to plan other than those four might feel the lack. In the plan part, "My training plan' is on top in bold, so it is noticeable and gives me information about what I need and what I am sufficient. (U6)

This is the app that is faithful to the basics of pedometer. The number of steps is on the very main in large letters. It is very easy to see calories and walking time. The system called 'accomplishment' gives fun to walk. (U10)

The start button is cumbersome and it is necessary to increase the reliability of information.

Other apps count steps automatically, but in this app, I have to push START button to count. Other apps have charged premium version that I can use with no advertisement, but this pedometer app only has free version. (U2)

It seems nice that it establishes exercise plan for each person's goal as a healthy app, but it might be more reliable if there is information about the trainer like photo or name. (U6)

\subsubsection{Interface aspect}

It is easy and intuitive to use and the contents are properly distributed on the screen.

This app is easy to understand and convenient to use because it is executed as soon as I input age, weight, height and gender. It checks the number of my steps and marks calories consumed while walking, distance and speed per hour, so I could know what to do even when I first used this app.(U1)

The menus are well organized, and information is displayed neatly. It has good statistics data, and the simple layout enables me to understand the operation method easily. (U3)

The app shows necessary information moderately not excessively. All the menus are displayed in icons that are easy to understand without explanation, so a lot of information can be on the first page. The app only has details about pedometer, no operation procedure or operation method. (U7)

The app is very convenient because it tells everything before searching information. The information is provided easily by touch on the intuitive interface. (U10)

The location of the advertisement is inconvenient, and it is necessary to increase readability.

Advertisements are attached together at the tap below, so I keep touching the advertisements by mistake. How about putting the advertisements on the top instead? (U10)

If it is displayed as "steps/targeted steps" instead of the targeted number of steps in small letters, it might be a lot better to see. (U6)

\subsection{FGI result of pedometer app users}

\subsubsection{Content aspect}




\section{Customized features that help users set and achieve their goals stand out, and are easy to use.}

It motivates me by telling how many steps do I need to reach the goal on the main page. It is interesting that it tells the total number of steps so far and how many steps I need to go around the earth. This is a perfect pedometer app to establish exercise goal alone. (U2)

Users can choose programs that they want in the walking plan, and the personal information section enables users to set up gender, step width and weight, so each user can enjoy customized exercise. Users can acquire intuitive information on the main page without needing to go to other menus. (U8)

It is convenient to establish a goal and to check what percentage I accomplish at a glance upon starting the app (U9)

This app seems to be faithful in the basic of pedometer. It is interesting that it shows the goal to accomplish at the top. (U10)

This app's great features are the overall intuitive interface and the information clearly shown on the main page. The category 'Challenge' stimulates the competitive spirit by enabling uses to engage in the challenge with other users or to challenge a race alone. There is a category that analyzes my steps, and the category is divided in card-layout, which is easy to see. In addition, the app analyzes in various ways, so it is very reliable. (U4)

There are no eye-catching special features, and naming is somewhat unintelligible.

This app is neat and pretty good, but there is no special feature, so it is not that impressive. (U3)

It might be easier to understand if the total distance of travel on the top of the main page is marked in simply km instead of difficult titles such as 'Roman Holiday' and 'Olympus Conquest'. (U6)

\subsubsection{Interface aspect}

The information is well classified, and the main screen provides enough information.

It shows graphs for each time frame as scrolling down, and at the bottom are menus horizontally. Therefore, it is easy to go to other menus at any pages. All the information necessary for pedometer app is on the main page. The detailed information can be seen at the calendar and report menus. The menu category is well divided with names, so it is easy to gain information that I want. (U2)

This app has good structure to comprehend accomplishments by showing steps, consumed calories and distance by date through the calendar. (U6)

Most information is located in the main page, so I didn't have to search separately. The top of the main page conveniently shows how many steps I need to accomplish the goal, and the center shows today's steps. The right top has "+" that enables to pause, stop and reset the pedometer, which is convenient. (U8)

It has a lot of information, but it is not easy to find.

The menu organization is neat and simple. The main page shows information that many people want to see, but I didn't know what to do for more detailed information. (U3)

It is a little inconvenient that I had to see graphs for each time frame and the items I input when I scroll on the main page. The items I input are covered by the banner advertisements, which is not convenient. (U2)

It is good that there are several categories, but it is difficult to find the categories since I had to click several links. (U7) 


\section{Conclusion and suggestions}

This study was intended to provide a guideline for sharing the in-depth experience of the step count app user and improving the satisfaction level and intention to use continuously.

For this, first, in order to continue and strengthen the use and motivation of the step count app, the level of play of users through the health app must be increased. The competition and reward system, sharing with friends through SNS, and personalized services have been shown to motivate users and motivate them to use the app.

Second, a step count app having various functions should be developed. Most of the currently available step count apps are easy to use, so there is no difficulty in using them, but they are faithful to the unique function of measuring the number of steps and provide only simple and similar functions between apps. Therefore, there is a need to induce interest by developing with differentiation by focusing on the needs of various users.

Finally, it appears that there is a risk of reducing the user's reliability due to inaccurate measurement of the automatic detection function, such as the measurement of the number of steps or exercise distance, so it seems that technical support from developers is necessary.

The result of this study is meaningful in that it provides practical guidelines that can lead to continuous use as well as content development of health apps that reflect users' information needs.

\section{Acknowledgements}

This research was supported by Basic Science Research Program through the National Research Foundation of Korea (NRF) funded by the Ministry of Education (NRF2018R1D1A3B07045595).

\section{References}

[1] Y. C. Jung, "Media usage behavior of 20 smart generations," KISDI STAT Report, vol.13, no.05-01, pp.1-8, (2013)

[2] Kenealy T. W., Parsons M. J., Rouse A. P., Doughty R. N., Sheridan N. F., Hindmarsh J. K., and Rea H. H., "Telecare for diabetes, CHF or COPD: Effect on Quality of Life, Hospital Use and Costs," A Randomised Controlled Trial and Qualitative Evaluation, PLoS One, vol.10, no.3, e0116188, (2015) DOI:10.1371/journal.pone.0116188 